\title{
Recovery of Slice Rotations with the Stack Alignment Transform in Cardiac MR Series
}

\author{
Constantine Zakkaroff ${ }^{1}$ \\ mnkz@leeds.ac.uk
}

Aleksandra Radjenovic ${ }^{2}$
a.radjenovic@leeds.ac.uk
John Greenwood ${ }^{3}$
j.greenwood@leeds.ac.uk
Derek Magee $^{1}$
d.r.magee@leeds.ac.uk

\author{
${ }^{1}$ School of Computing \\ University of Leeds \\ Leeds, LS2 9JT, UK \\ ${ }^{2}$ NIHR Leeds Musculoskeletal \\ Biomedical Research Unit (MBRU) \\ University of Leeds \\ Leeds, LS7 4SA, UK \\ ${ }^{3}$ Leeds Institute of Genetics, \\ Health and Therapeutics (LIGHT) \\ University of Leeds \\ Leeds, LS2 9JT, UK
}

\begin{abstract}
Generic solutions for misalignment correction in image stacks are based on slice-byslice registration to a reference volume. Slice misalignment in cardiac Magnetic Resonance cine series typically includes translational and rotational components. Recovery of rotation in medial down to apical slices with the basic slice-to-volume registration may provide unreliable results because of rotational symmetry of the short-axis view of the left ventricle. This paper describes a novel method for correction of stack misalignment in cardiac cine series with recovery of the rotational component. The core of the presented method is a custom spatial transform which parametrises a set of independent centred rigid transforms, one per slice in a stack. The stack alignment transform was designed to circumvent the weaknesses associated with slice-to-volume registration and improve the reliability of misalignment correction over the slice-by-slice registration approach. The image similarity metric for every iteration during the stack alignment transform optimisation is calculated on the whole image stack with all slice correction parameters contributing to the result. The presented method was evaluated on 50 clinical datasets and the results demonstrate an improvement of misalignment correction with the proposed transform. The study also provides evidence of the importance of recovery of slice rotations in this application; the presented method is general enough to be applied to similar problems in other image modalities.
\end{abstract}

\section{Introduction}

Displacement of individual slices in image stacks relative to each other is known as stack misalignment and can occur in imaging modalities with discontinuous acquisition protocols. This type of error can be hard to notice upon visual examination short of reformatting the stacks to be displayed in orientations other than the initial imaging plane. A failure to compensate for the individual slice movement undermines the accuracy of the subsequent image 
analysis. Stack misalignment in cardiac Magnetic Resonance (MR) cine series reduces the reproducibility of volumetric measurements computed for diagnostic purposes. Wall motion and volumetric measurements for the assessment of myocardial function are derived from cine stacks spanning the cardiac cycle. During image acquisition breath-holding is used to exclude respiratory motion; a patient holds their breath multiple times and one to two slices over all cardiac phases are acquired during a breath-hold. The misalignment is caused by inconsistencies in breath-hold positions between slice acquisitions and depends on the patient's ability to follow the instructions of the attending technician during the scan.

\subsection{Misalignment Correction Background}

As with most registration applications the solutions to the problem of slice misalignment in image stacks rely on a reference image, which provides the criteria for movement compensation during transform optimisation. Slice displacements in cardiac cine stacks typically include translational and rotational components. Recovery of rotation in medial down to apical slices with the basic slice-to-volume registration may provide unreliable results because of rotational symmetry of she short-axis (SA) view of the left ventricle (LV).

A solution which uses long-axis (LA) cine series as the reference image for SA cine stack correction with a 2D translation transform for each slice provides reliable results [ $\square$ ]; the use of 4D (3D + time) images in the method enhances its appeal, but it is only feasible if the LA series are multi-slice series; this is not the case in the study presented in this paper. Another solution relies on the slice-to-volume registration of SA slices to a high-resolution reference volume $[\square]$; the multi-resolution registration optimises a 3D rigid transform for each SA slice from end-diastolic phase as it is registered to the 3D whole heart volume. The results of stack correction experiments with slice-to-volume registration show the improvement in the accuracy for ventricular function analysis, although the study was performed on a relatively small number of healthy volunteers. Furthermore, as the authors in [四] observe, slice-tovolume registration can become an under-constrained problem due to the method's weakness which lies in the use of out-of-plane rotations.

\subsection{Stack Alignment Transform Overview}

The stack alignment transform combines the individual slice transforms into one parameter space so that all transforms can be optimised simultaneously. While at first it might appear that the simultaneous optimisation does not provide any advantages over slice-by-slice transform optimisation, in reality this solution makes a better use of the image similarity metric by increasing the accuracy of the metric value calculation. Consider the case of slice-to-volume registration where the Mutual Information (MI) image similarity metric is evaluated from the region of overlap of a single slice in a stack with a 3D volume: the joint probability distribution is likely to be a poor representation of the true joint probability distribution. However, a much better representation of the joint probability can be obtained from the whole stack and the reference volume; in this case the joint histograms which are used in the calculation of the MI value would contain a larger number of co-occurrences of grey-levels which represent the types of tissue in the images. This method of MI optimisation is possible with a custom stack alignment transform presented and evaluated in this paper; the transform combines the parameters for a set of rigid centred 2D transforms which define the translation and rotation for individual slices. 
The stack alignment transform deliberately avoids out-of-plane translations and rotations which otherwise pose a problem for the reconstruction of the corrected series: both out-of-plane translations and rotations may result in the "holes" in the reconstructed series. Respiration-induced motion has been estimated to introduce up to $23 \mathrm{~mm}$ total displacement of the heart due to patients' inconsistency in breath-hold positions [ $[$ ], which is likely to shift individual slices past their in-plane positions. The authors in [ []$]$ suggest the use of linear interpolation between slices in the series to fill the gaps to restore the integrity of the image. However, the reconstructions with linear interpolation between non-adjacent stack slices degrade the edges and other features in the image.

The stack alignment transform has been developed for an application of spatiotemporal registration of angiography and perfusion data described in $[\square]$; this application is aimed at the creation of patient-specific maps which co-locate perfusion defects observable in perfusion series with coronary arteries imaged in angiography volumes. The major branches of coronary vascular trees usually run roughly along the direction of the LA rather than along the SA planes; for the co-location of perfusion defects with coronary trees the rotational component of contractile motion is more important than the out-of-plane motion. From this point of view it is reasonable to constrain the slice displacements during misalignment correction to in-plane motion in order to avoid the loss of image features due to out-of-plane slice displacements (and subsequent interpolation) at the expense of some out-of-plane error. Hence, the main requirement imposed on the stack alignment transform is to preserve image features in the cine slices in favour of recovering the finer components of myocardial motion such as radial rotation around the LA of the heart.

\section{Materials and Methods}

The data in this study were acquired on a dedicated cardiac research scanner (1.5 Tesla Intera CV, Philips, Best, The Netherlands) during a comprehensive trial on MR imaging in coronary heart disease, CE-MARC [ $⿴ 囗$ ]; the experiments involved two types of cardiac data:

Coronary MR angiography: Three dimensional whole heart coronary MR angiography acquired using a balanced SSFP sequence and a respiratory navigator to compensate for respiratory motion during free breathing; timing of the diastolic rest period was estimated from the four-chamber free breathing cine scan, with the duration of acquisition up to $120 \mathrm{~ms}$ per R-R interval (determined by length of diastolic rest period), matrix of $304 \times 304$, field of view 320-460 mm, slice thickness $0.9 \mathrm{~mm}, 100-120$ slices as required.

Resting wall-motion: Contiguous cine stack encompassing the entire LV in 10-12 slices (depending on left ventricular LA length) acquired during multiple breath-holds, with the matrix of $192 \times 192$, field of view $320-460 \mathrm{~mm}$, slice thickness $10 \mathrm{~mm}$, at least 20 phases per cardiac cycle, 1-2 slices per breath-hold.

Further information on the pulse sequences in the CE-MARC trial is available in [ $Q$ ]. In this study 50 representative datasets from the CE-MARC trial were selected randomly by the clinical trials unit; one dataset was excluded because of the severe motion artefacts in the angiography volume.

\subsection{Pre-registration and Cine Phase Selection}

Prior to the optimisation of the stack alignment transform it is necessary to find the cardiac phase in the cine series that corresponds to the reference angiography volume acquired at 


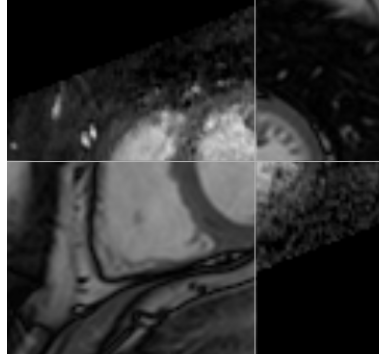

(a) Cine and reference original

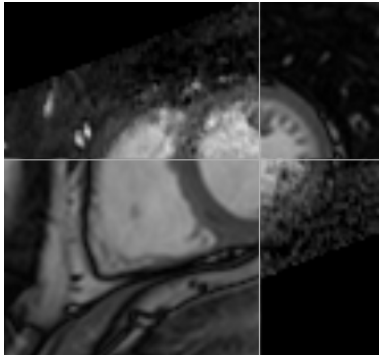

(b) Cine and reference corrected

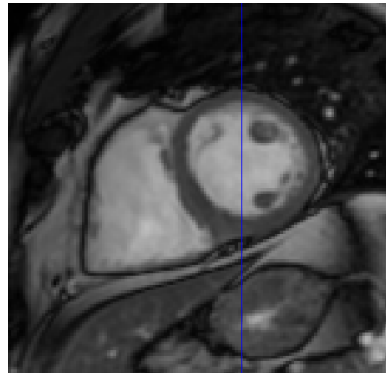

(c) Slicing plane indication

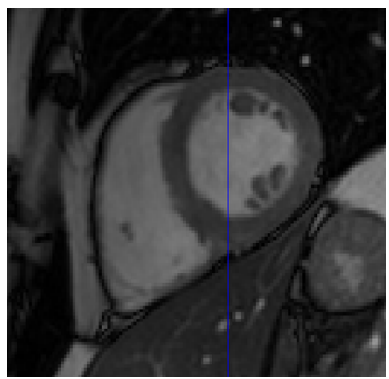

(f) Slicing plane indication

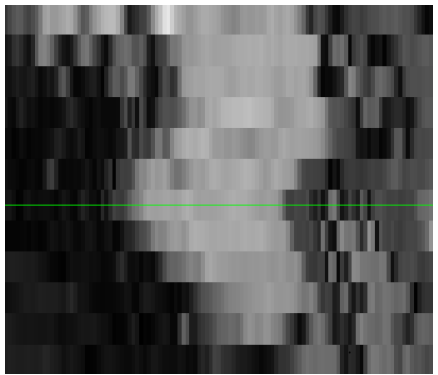

(d) LV before correction

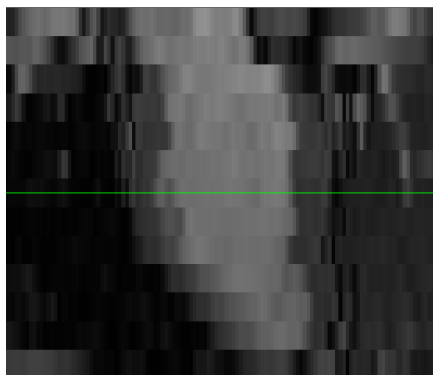

(g) LV before correction

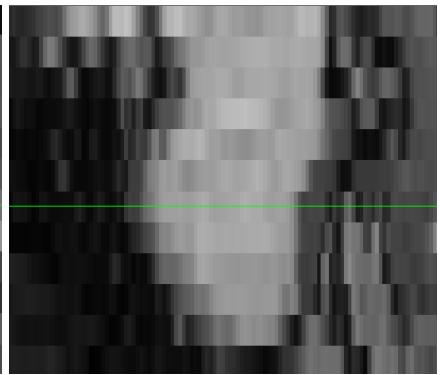

(e) LV after correction

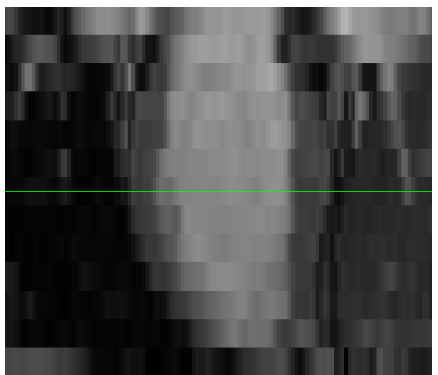

(h) LV after correction

Figure 1: Misalignment correction examples: checkerboards (top) show the alignment of a single SA slice from a cine stack and double-oblique reformatted angiography volume before and after correction; images in the second and third rows show two cine stacks in LA orientation (location of the slicing plane is indicated in the left-most images in each row) before and after correction.

end-diastolic phase. Although either visual examination of the cine series or ECG trigger time normalisation can be used to determine the matching phase, this study uses MI-based phase match determined by the best MI value computed for all cine phases and the angiography volume during the pre-registration step with 3D translation transform. Although it would be just as easy to optimise a rigid 3D transform with translation and rotation instead of a translation-only transform, it was deliberately avoided because theoretically there can be multiple cases of stack misalignment producing an appearance of 3D rotations. An example of such misalignment is shown in the bottom row in Figure 1: the registration of the misaligned image in $1(\mathrm{~g})$ to the corresponding reference volume would likely result in a $3 \mathrm{D}$ 
rotation of the whole image stack. Hence it is preferable to use the simplest possible transform in order to avoid 3D rotational errors. The pre-registration method in this case is a straightforward example of volume-to-volume registration with a $3 \mathrm{D}$ translation transform between a given cine phase and angiography volume.

A set of elliptic masks derived from manually-defined rectangular regions of interest (one for each slice) in the end-diastolic phase of the cine was used to exclude irrelevant anatomical features. Multi-resolution registration (two levels) with a Regular Step Gradient Descent optimiser and Matte's implementation of MI [ब] available in ITK [0] provided fast and reliable results. In practice, the best match usually falls in a window of \pm 2 phases from the normalised ECG trigger time of the angiography volume.

\subsection{Stack Alignment Transform Parametrisation and Optimisation}

The stack alignment transform parametrises separately the in-plane translation in physical space units along the $X$ and $Y$ dimensions and rotation in radians around a user-supplied centre of rotation for the individual slices, independent of each other. In addition, the transform includes a parameter for global translation along the $Z$ direction. For example, for a series of $N$ slices (typically in the range of 10 to 14 slices in this study) the transform can be represented as parameter vector $V=\left\{\left\{\theta_{1}, C_{x 1}, C_{y 1}, T_{x 1}, T_{y 1}\right\}, \ldots,\left\{\theta_{N}, C_{x N}, C_{y N}, T_{x N}, T_{y N}\right\}, T_{z}\right\}$. The transform also includes a set of fixed parameters, which are used to calculate the relevant slice number and the corresponding subset of transform parameters when given a point in 3D space. Fixed parameters include image origin, size, and voxel spacing, all obtained from the corresponding cine phase. Given a point $P=[x, y, z]$ in $3 \mathrm{D}$ space, the transformed point $P^{\prime}=\left[x^{\prime}, y^{\prime}, z^{\prime}\right]$ is calculated in two steps as follows:

$$
\left[\begin{array}{c}
x^{\prime} \\
y^{\prime}
\end{array}\right]=\left[\begin{array}{cc}
\cos \theta_{n} & -\sin \theta_{n} \\
\sin \theta_{n} & \cos \theta_{n}
\end{array}\right] \cdot\left[\begin{array}{c}
x-C_{x n} \\
y-C_{y n}
\end{array}\right]+\left[\begin{array}{c}
T_{x n}+C_{x n} \\
T_{y n}+C_{y n}
\end{array}\right], z^{\prime}=z+T_{z}
$$

where $n$ is an index into the $Z$ dimension on the image grid, identifying the number of the slice, which point $P$ falls into; the index is computed from the fixed parameters of the transform. The centre of rotation parameters $C_{x n}$ and $C_{y n}$ in each case were initialised to the centre of the image mask for a given slice. Apart from the stack alignment transform, the rest of the registration components for this stage are the same as for the pre-registration: multiresolution pyramids (two levels), Regular Step Gradient Descent optimiser, Matte's MI and cine image masks. During the registration the cine stack is used as the fixed image, because the image similarity metric collects samples for calculating image gradients and derivatives from the moving image on the fixed image grid. After the registration the inverted stack alignment transform can be applied to correct/resample all phases in the cine series.

\subsection{Evaluation Protocol}

The performance of correction parameter recovery was evaluated for convergence reliability and accuracy. First our method was evaluated with a protocol of registration uncertainty measurement described in [س]. The protocol is designed for assessing the stability of registration in a large number of runs, each with a known introduced misalignment; in this evaluation method the mean recovered transform is used as a pseudo-gold standard based on the assumption of a zero mean distribution of errors. In our study 100 random simulated misalignments for each slice in a given dataset were generated; the in-plane rotation 
parameters $\Theta_{M}=\left\{\theta_{M 1}, \ldots, \theta_{M 100}\right\}$ were restricted to \pm 4 degrees; the in-plane translations $X_{M}=\left\{x_{M 1}, \ldots, x_{M 100}\right\}$ and $Y_{M}=\left\{y_{M 1}, \ldots, y_{M 100}\right\}$ were restricted to $\pm 4 \mathrm{~mm}$. This type of simulated slice displacement is significantly more severe than the misalignment common for clinical datasets, because typically only a small portion of slices in a single dataset contains observable movement, while the simulated transforms shift every slice out of its place. During the evaluation, a clinical dataset with an unknown initial error was repeatedly further misaligned with one of the 100 simulated displacements. The recovered in-plane displacement for each slice after the registration can be represented as $\Theta_{R}=\left\{\theta_{R 1}, \ldots, \theta_{R 100}\right\}$, $X_{R}=\left\{x_{R 1}, \ldots, x_{R 100}\right\}$ and $Y_{R}=\left\{y_{R 1}, \ldots, y_{R 100}\right\}$; the estimated correction parameters for a given slice are represented as $\Theta=\Theta_{M}+\Theta_{R}, X=X_{M}+X_{R}$ and $Y=Y_{M}+Y_{R}$, while the arithmetic means $\bar{\Theta}, \bar{X}$ and $\bar{Y}$ represent the mean transform. In practice the displacement of the centre of rotation parameters $C_{x n}$ and $C_{y n}$ (as defined in equation (1)) for any given slice was small and thus it was not considered in the evaluation. The magnitude of rotational and translational errors $R_{e r r}$ and $T_{e r r}$ in a single experiment $n$ can be calculated as follows:

$$
\begin{gathered}
R_{e r r}(n)=\left\|\theta_{n}-\bar{\Theta}\right\| \\
T_{e r r}(n)=\sqrt{\left(x_{n}-\bar{X}\right)^{2}+\left(y_{n}-\bar{Y}\right)^{2}}
\end{gathered}
$$

The second part of evaluation involved the measurement of registration accuracy from manually-defined contours treated as the gold-standard. In addition, this part of the evaluation was aimed at providing evidence to support the case for recovering the rotational component of slice displacement. Three contours (LV endocardial, LV epicardial and rightventricular (RV) endocardial) were manually defined for a subset of patients including 24 datasets; the cine contours were defined in one of the basal slices in the end-diastolic cine phase determined by the pre-registration; the reference contours were defined in the slices extracted from the reformatted pre-registered reference volumes. The accuracy of contour match was calculated as the Hausdorff distance metric, which is used for determining the degree of similarity between two objects when superimposed on one another [曰]; Hausdorff distance is defined as follows:

$$
H(A, B)=\max (h(A, B), h(B, A))
$$

where

$$
h(A, B)=\max _{a \in A} \min _{b \in B}\|a-b\|
$$

is the directed Hausdorff distance and $A$ and $B$ are contours in the input images.

Hausdorff distance was calculated for cine and reference contours before misalignment correction, after correction with translation, and after correction with translation and rotation. The results for both parts of the evaluation are summarised in the following section.

\section{Results}

Visual inspection of the registration results with checkerboards combining the reference and corrected cine images for the 49 datasets without simulated errors showed that misalignment was recovered successfully in all cases. Figure 1 presents three examples of misalignment and its correction typical for the results in this study. It should be noted that the checkerboard method for visual registration validation is not specific enough for assessing the accuracy of 

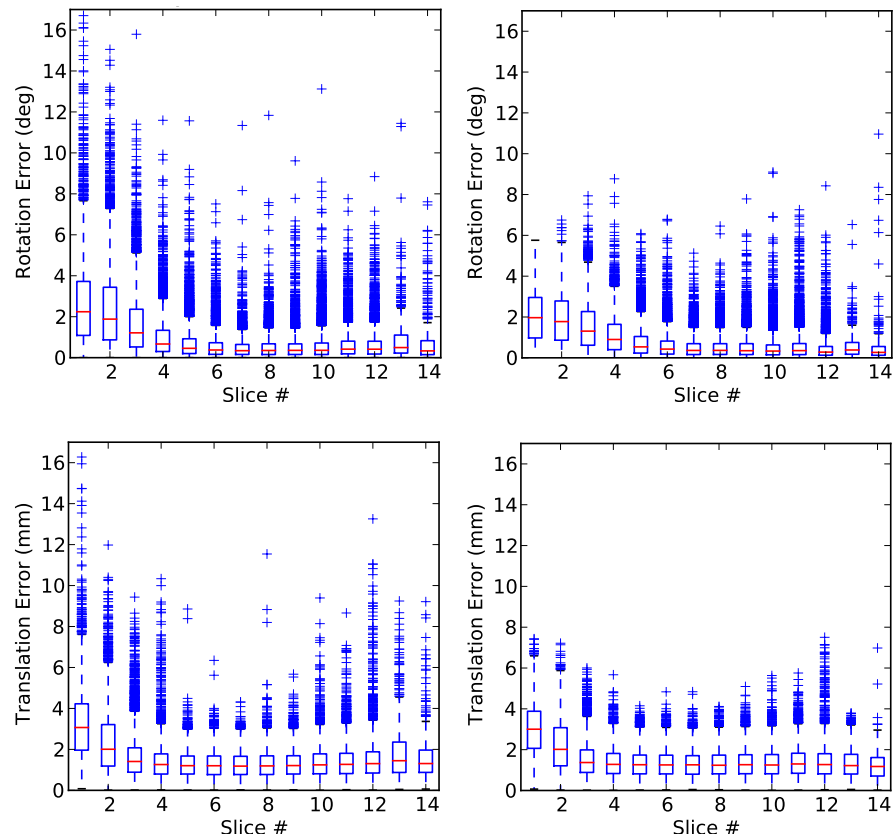

Figure 2: Distribution of errors $R_{e r r}$ (top row) and $T_{e r r}$ (bottom row) as defined in equations (2) and (3); left: slice-by-slice correction, rotation and translation; right: stack alignment transform correction, rotation and translation; the box plots summarise the errors for a 100 simulated runs for all datasets, one bar per single slice index in a stack.

rotation recovery. Most datasets contained visually-detectable correction for a small subset of slices (usually one to three) while a few datasets required correction for all slices. The LA-reformatted cine images of LV in 1(d) and 1(e) show the cases of strong misalignment where most slices required visually-detectable correction.

Parameter recovery after the application of simulated misalignment described in the previous section was compared against the slice-by-slice registration restricted to in-plane translation and rotation. Figure 2 shows the comparison of the distributions of the rotation and translation errors $R_{e r r}$ and $T_{\text {err }}$ (as defined in the equations (2) and (3)) for the 49 datasets; correction with the stack alignment transform features a more stable and accurate performance for both rotation and translation displacement components. The higher error ranges for the extreme apical and ventricular slices are explained by: (a) insufficient image features (e.g. no clearly visible myocardial/ventricular walls) or (b) insufficient image overlap region between apical or ventricular slices in the cine and angiography. For the slices with clearly visible myocardium the recovered rotation and translation errors peak around 0.3 degrees and one voxel (1.5 mm approximately) respectively and are bounded by 1.5 degrees and 2.5 $\mathrm{mm}$ in $95 \%$ of cases for both slice-by-slice alignment and stack alignment transform experiments. However the difference between the overall shape of the error distributions shows that our method is more consistent in a wider range of slices and has a greater capture range.

A pair of examples from the gold-standard evaluation is shown in Figure 3. In the top row example the translation-only transform does improve the contour overlap, but rotation recovery undoubtedly provides a better result in this case; in the bottom row example the contours 


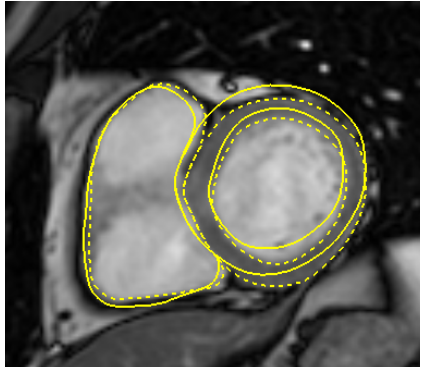

(a) Before correction

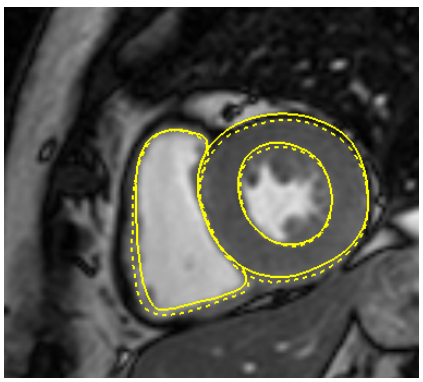

(d) Before correction

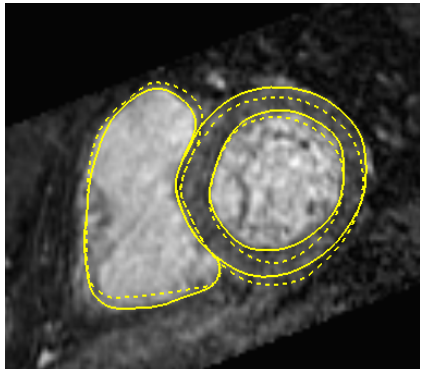

(b) After translation

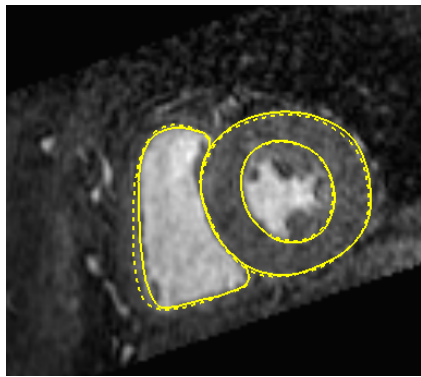

(e) After translation

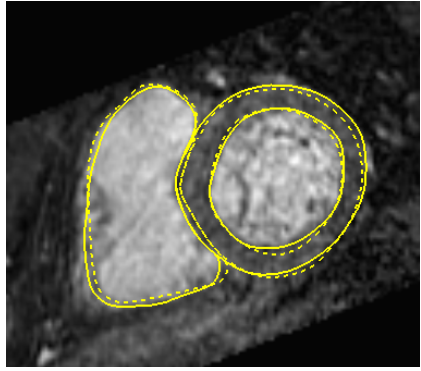

(c) After rotation and translation

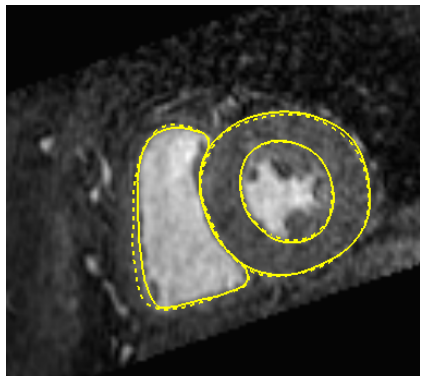

(f) After rotation and translation

Figure 3: Left: cine slice and contours (dashed line) with reference contours (solid line) as defined in reformatted angiography and superimposed on the cine contours without registration; centre: cine contours after translation-only correction, superimposed on the reference contours in the reference volume; right: cine contours after rotation and translation correction, superimposed on reference contours in the reference volume.

show that the use of transform with rotation does not improve significantly the accuracy of correction. In practice many datasets can be successfully corrected with translation-only registration, but up to $25 \%$ datasets in this study required rotational correction for satisfactory results. The paired Wilcoxon test for non-parametrict distributions confirmed $(p<0.05)$ that the recovery of slice rotation component significantly improved the Hausdorff distance metric for LV endocardial and LV epicardial contours; the RV endocardial contours did not show significant improvement with rotation recovery most likely due to the ambiguity of the $\mathrm{RV}$ endocardial boundary and the resulting subjectivity of manual contour deliniation, which is discussed in detail shortly.

The Hausdorff distance metric bar charts in Figure 4 provide a further insight into misalignment correction with the stack alignment transform. Again the bar charts show that in general a reasonable improvement in Hausdorff distance can be achieved with translationonly correction, however in about a quarter of datasets rotation recovery provides significant improvement. The results for the LV endocardial contours show the most consistent improvement over all datasets because the LV endocardial contours in cardiac MR cine series usually are easy to identify: the clear blood-to-myocardium interface along with the simple shape of the LV make it a simple target for manual segmentation. The LV epicardial contours pose a more ambiguous task for ground-truth segmentation; the RV endocardial contours are the hardest to define because of the complex shape of the RV and thin myocardium. However during registration the image features of the RV are crucial for recovering slice rotations. 

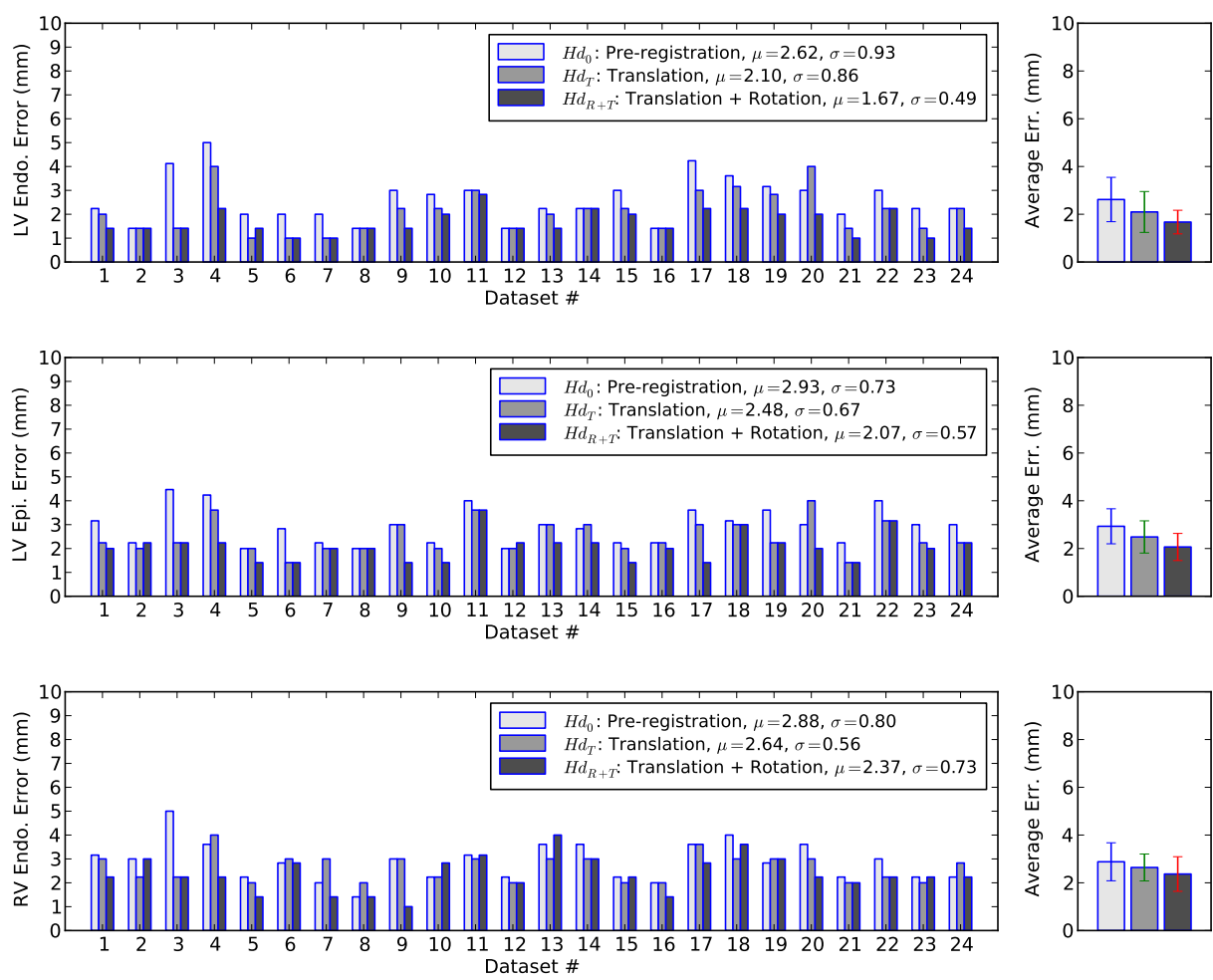

Figure 4: Hausdorff distance metric for LV endocardial (top), LV epicardial (middle) and LV endocardial (bottom) contours; the metric values are compared for contours before correction, after translation-only correction, and after correction with rotation and translation; the plots on the right summarise the Hausdorff distance values for the corresponding contours.

\section{Discussion and Future Work}

In this paper we presented a custom transform for recovery of rotational and translational components of stack misalignment in cardiac MR series; the transform is designed to overcome the difficulties associated with slice-to-volume registration which sometimes may become an under-constrained problem. The stack alignment transform fulfils its purpose by preserving the inter-slice offsets and co-planar orientation of the slices; these constraints favour the integrity of the cine series and are intended to avoid gaps and slice overlaps in the corrected series. The transform also offers a reliable method of optimising the MI image similarity metric by enabling the computation of the metric value for the whole image stack instead of just the region of overlap of a single slice with the reference volume; the correction parameters for all slices are considered in every optimisation iteration thus providing a means for calculating a robust estimation for joint probability distributions in the reference and cine images. The evaluation of registration with stack alignment transform against the basic slice-by-slice registration provides evidence that the transform improves the robustness of misalignment correction.

In future work we plan to extend the stack alignment transform with an additional parameter for the global translation in the fourth (temporal) dimension. This would allow us to 
avoid the explicit step of phase selection during pre-registration. Furthermore, the accuracy of the implicit (in this case) phase-selection would be improved because the optimal phase match would be determined gradually along with the process of optimising the positions of all slices in the series. However the pre-registration step cannot be avoided entirely because of the possibility of large differences in the patient's position occurring as a result of motion between the scans. Similar custom transforms combining in-plane rotations with 4D translations are suitable for other types of spatiotemporal registration, e.g. the registration of cardiac perfusion series with the cine series.

\section{Acknowledgements}

Clinical data were obtained within the CE-MARC study funded by the British Heart Foundation Grant to Leeds CMR Unit (grant RG/05/004-J.G., A.R.). This work was funded by the Top Achiever Doctoral Scholarship awarded by Tertiary Education Commission of New Zealand (grant UOLX08001-C.Z.) and WELMEC, a Centre of Excellence in Medical Engineering funded by the Wellcome Trust and EPSRC (grant WT 088908/Z/09/Z-A.R., D.M.).

\section{References}

[1] W Birkfellner, M Figl, J Kettenbach, J Hummel, P Homolka, R Schernthaner, T Nau, and H Bergmann. Rigid 2D/3D Slice-to-volume Registration and its Application on Fluoroscopic CT Images. Medical Physics, 34(1):246-255, 2007.

[2] A Chandler, R Pinder, T Netsch, J A Schnabel, D J Hawkes, D L G Hill, and R Razavi. Correction of Misaligned Slices in Multi-slice Cardiovascular Magnetic Resonance Using Slice-to-volume Registration. Magnetic Resonance Imaging, 10(1):13, 2008.

[3] J P Greenwood, N Maredia, A Radjenovic, J M Brown, J Nixon, A J Farrin, C Dickinson, J F Younger, J P Ridgway, M Sculpher, S G Ball, and S Plein. Clinical Evaluation of Magnetic Resonance Imaging in Coronary Heart Disease: The CE-MARC Study. Trials, 10(1):62-71, 2009.

[4] John P Greenwood, Neil Maredia, John F Younger, Julia M Brown, Jane Nixon, Colin C Everett, Petra Bijsterveld, John P Ridgway, Aleksandra Radjenovic, Catherine J Dickinson, Stephen G Ball, and Sven Plein. Cardiovascular Magnetic Resonance and Single-photon Emission Computed Tomography for Diagnosis of Coronary Heart Disease (CE-MARC): A Prospective Trial. Lancet, 379(9814):453-60, 2012.

[5] D P Huttenlocher, G A Klanderman, and W J Rucklidge. Comparing images using the Hausdorff distance. IEEE Transactions on Pattern Analysis and Machine Intelligence, 15(9):850-863, 1993.

[6] L Ibáñez, W Schroeder, L Ng, and J Cates. The ITK Software Guide, 2nd edition, 2005.

[7] J Lötjönen, M Pollari, S Kivistö, and K Lauerma. Correction of Movement Artefacts from 4-D Cardiac Short- and Long-axis MR Data. In Medical Image Computing and Computer Assisted Intervention, 2004. 
[8] D Mattes, D R Haynor, H Vesselle, T K Lewellyn, and W Eubank. Nonrigid Multimodality Image Registration. In Medical Imaging: Image Processing, volume 4322 of SPIE Conference Series, pages 1609-1620, 2001.

[9] K McLeish, D L G Hill, D Atkinson, J M Blackall, and R Razavi. A Study of the Motion and Deformation of the Heart Due to Respiration. IEEE Transactions on Medical Imaging, 21(9):1142-50, 2002.

[10] J R Sykes, D S Brettle, D R Magee, and D I Thwaites. Investigation of Uncertainties in Image Registration of Cone Beam CT to CT on an Image-guided Radiotherapy System. Physics in Medicine and Biology, 54(24):7263-83, 2009.

[11] C Zakkaroff, D Magee, A Radjenovic, and R Boyle. Mediated Spatiotemporal Fusion of Multiple Cardiac Magnetic Resonance Datasets for Patient-specific Perfusion Analysis. In Computing in Cardiology, volume 37, pages 469-472, 2010. 